\title{
A case of fracture talus with medial malleolus treated with headless screws and cancellous cannulated screws using single anteromedial approach
}

\author{
Shubham N. Katti*, Prithviraj A. Paigude, Praful A. Ingale, Sushilkumar R. Mane
}

Department of Orthopaedics, BJ Government Medical College and Sassoon General Hospital, Pune, Maharashtra, India

Received: 09 December 2021

Revised: 23 December 2021

Accepted: 24 December 2021

\section{*Correspondence:}

Dr. Shubham N. Katti,

E-mail: shubhamkatti@gmail.com

Copyright: () the author(s), publisher and licensee Medip Academy. This is an open-access article distributed under the terms of the Creative Commons Attribution Non-Commercial License, which permits unrestricted non-commercial use, distribution, and reproduction in any medium, provided the original work is properly cited.

\begin{abstract}
Talar fractures are complex injuries with an array of management options and complications. We present a case of talar fracture with medial malleolus fracture fixed via common anteromedial approach through the fracture site. The incidence of associated malleolar injury has ranged from $19 \%$ to $28 \%$ in prior studies. The skin condition is often poor and prone to swelling in such cases which makes the management challenging. Along with poor wound healing, joint stiffness, osteonecrosis and osteoarthritis of the ankle are complications affecting the outcome of the patient.
\end{abstract}

Keywords: Talus fracture, Medial malleolus, Osteonecrosis, Neck of talus

\section{INTRODUCTION}

Talus fractures are the second most common injuries of the tarsal bones. The incidence of associated malleolar injury has ranged from $19 \%$ to $28 \%$ in prior studies. ${ }^{1}$

In patients with displaced talus fractures, 90\% develop post-traumatic hindfoot arthrosis. ${ }^{2}$ Injuries associated with medial malleolar fracture are less likely to develop avascular necrosis (AVN). ${ }^{3}$ Fractures of the malleolus also aid in approaching the talar fracture and in reducing it, decreasing the need for any osteotomy. We present our experience in dealing with the management and outcome of such a case.

\section{CASE REPORT}

25-Year-old male presented to the emergency room with history of motor vehicle accident.

Complained of swelling, inability to bear weight on his left leg. Physical examination showed ecchymosis, swelling and tenderness along with painful restriction of movements. Distal neurovascular status examination was normal. Patient was promptly stabilized with short leg below knee slab and limb elevation.

\section{Management}

Standard AP and lateral views were obtained. A CT scan was performed. They showed a left sided Hawkins type III talar neck communited fracture, with posterior process fracture with fracture of medial malleolus.

Patient was operated within 12 hours. Anteromedial approach was utilized. The incision was made medial to the talar neck and medial to the anterior tibial tendon. Thick subcutaneous and periosteal flaps were elevated. A Denham pin was inserted in the calcaneum to assist in traction and reduction. Medial malleolar fracture fragment was retracted to allow exposure to the talus. Fracture was reduced provisionally using $\mathrm{K}$ wires. Reduction was confirmed in AP, lateral and canale views. 2 headless screws were then passed over guide wires. The medial 
malleolus was then reduced and fixed with 2 cancellous cannulated screws. Patient was given a below knee slab and advised non weight bearing for 2 months. Patient was followed up for up to 6 months to see improvement in function. Following was improvement in functional outcome of the patient as measured by the American foot and ankle society at 2, 4 and 6 months. ${ }^{4}$ At the end of 6 months patient was able to do all day to activities as well as engage in his occupation without any discomfort.

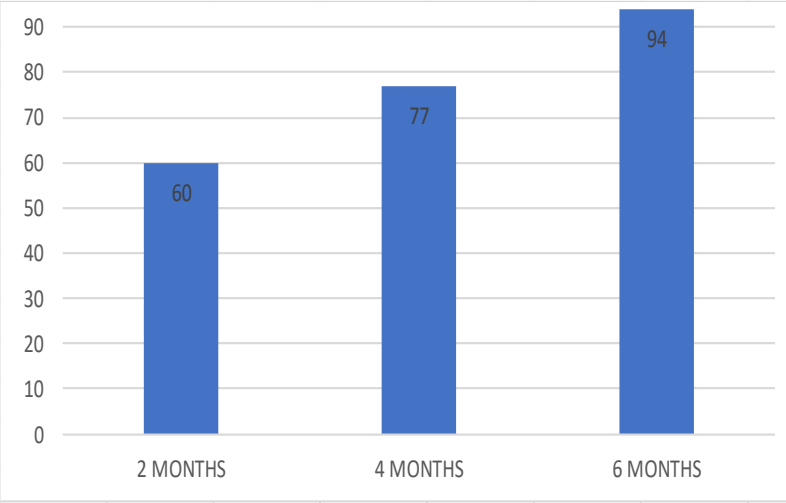

Figure 1: American foot and ankle society score at 2, 4 and 6 months.

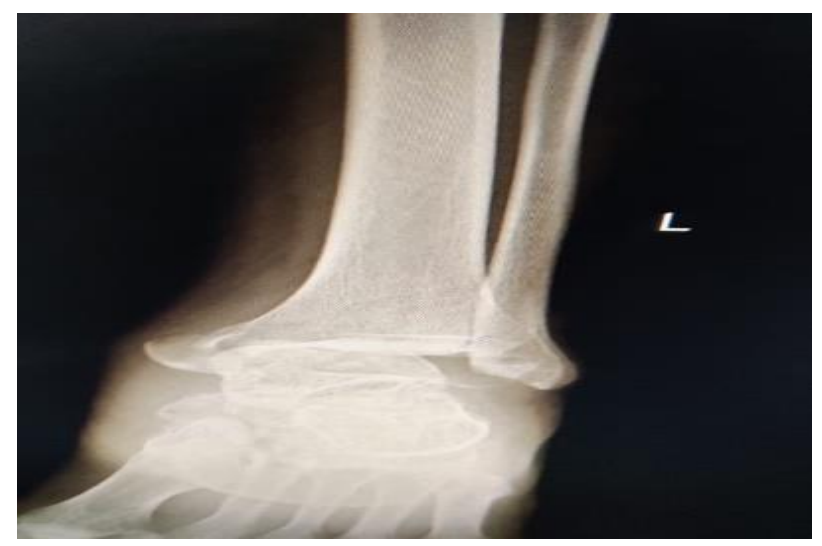

Figure 2: Preoperative $x$ rays ap view.

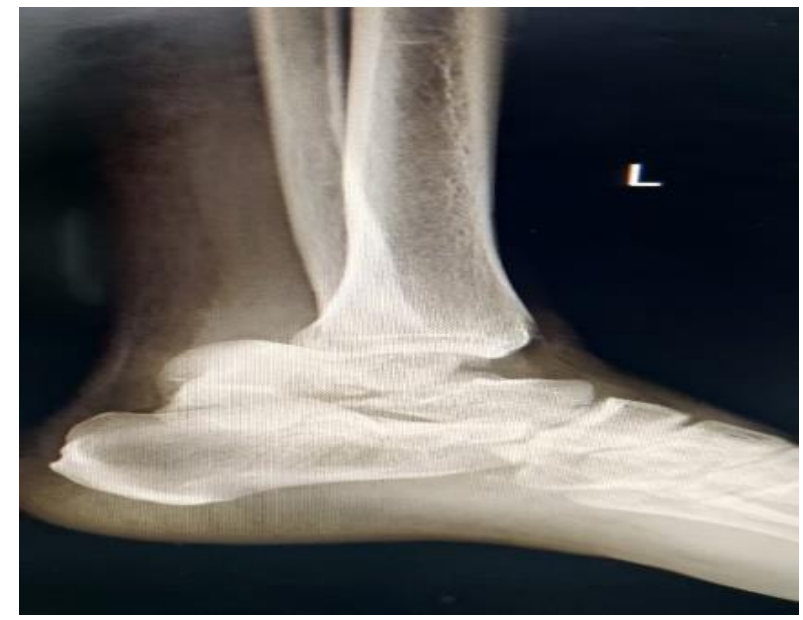

Figure 3: Preoperative x-rays lateral view.

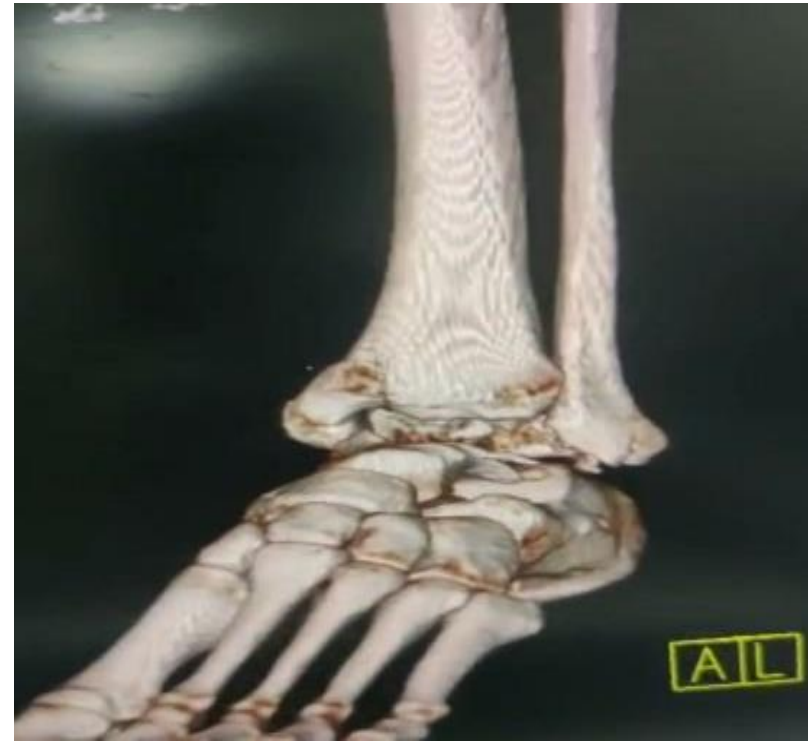

Figure 4: Preoperative CT scan.

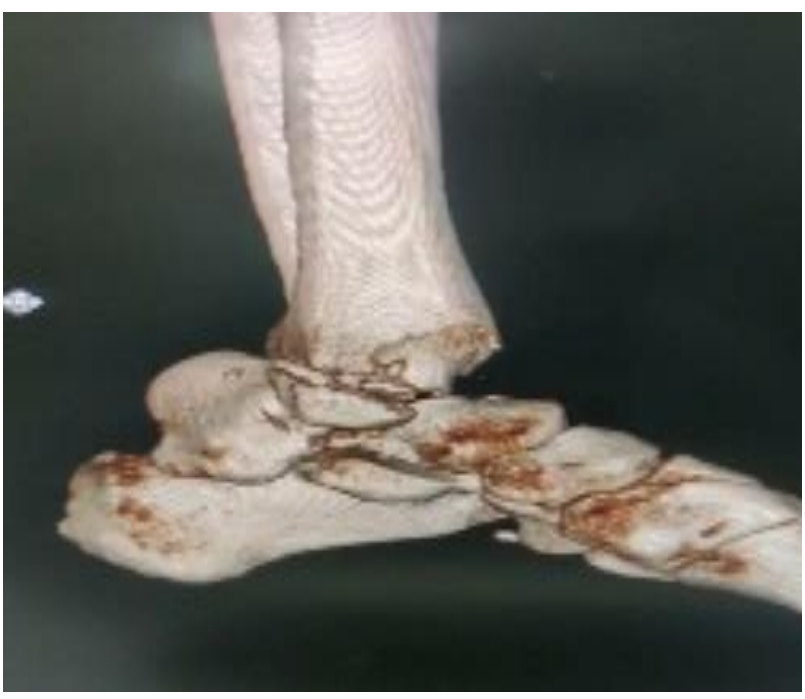

Figure 5: Preoperative CT scan.

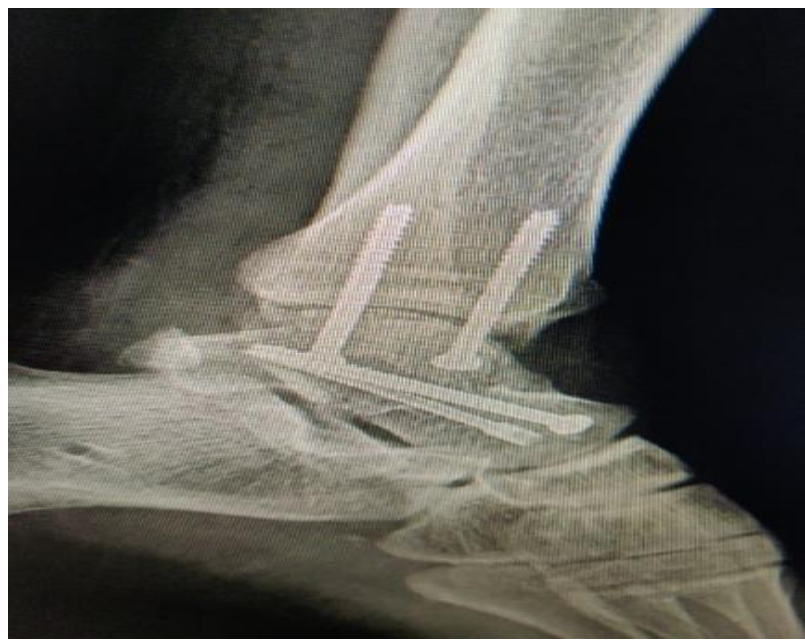

Figure 6: Post operative $x$ - ray o fracture reduction and fixation. 


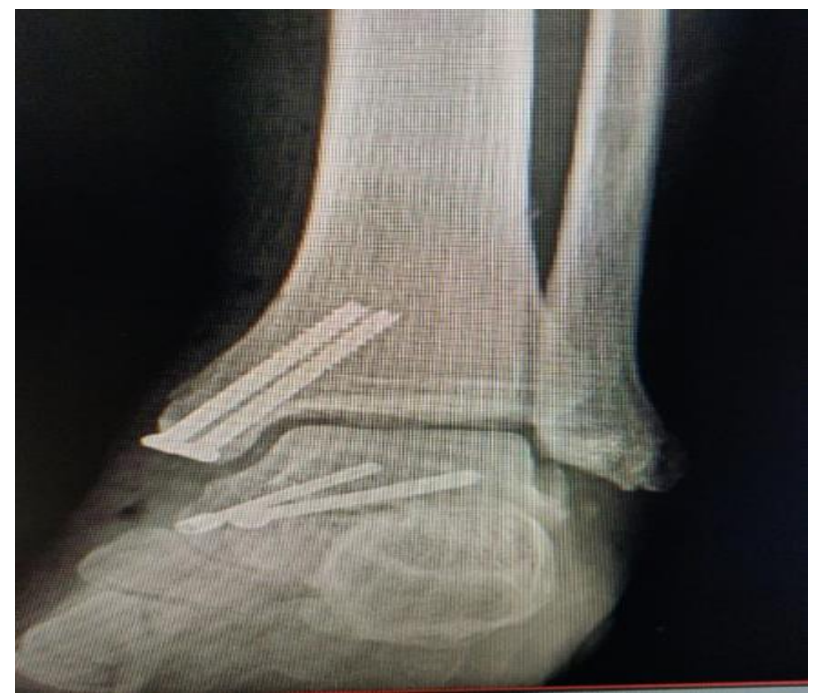

Figure 6: Post operative x-ray of fracture reduction and fixation

\section{DISCUSSION}

The management of talar fractures is very challenging with variable outcomes. The talus has over half its surface with no muscular attachment. The neck of the talus acts as a site of vascular flow predominantly at the dorsal neck. ${ }^{5}$

Amongst talar fractures, fractures of the neck account for up to $50 \%$ of all talar fractures. ${ }^{6}$ The timing of the surgery remains controversial. Recent studies contradict the previous notion that timing affects the risk of future osteonecrosis. Other factors like fracture communition, soft tissue trauma have been found to be significant. ${ }^{7,8}$ However, the given lack of clear guidelines on the subject we believe that displaced fractures should be taken for reduction and fixation on an emergency basis. We also operated our case within 12 hours of emergency room presentation.

The anteromedial approach along with the anterolateral approach is required commonly for proper visualization and reduction of the fracture. Many cases will also require a medial malleolar osteotomy to aid in visualization and reduction. ${ }^{9,10}$ The presence of associated medial malleolus fracture when present can aid in the visualization and reduction of the fracture. The anteromedial approach can help in the management of both the fractures and the visualization through the medial malleolar fracture site may obviate the need for another anterolateral skin incision. This can be particularly useful given the poor healing character of this region. The talus fracture is then fixed with two headless compression screws which serve the function of compression as well as anti-rotation function. The medial malleolus maybe fixed with either a ss wire and $\mathrm{k}$ wires or cancellous cannulated screws. Cancellous cannulated screws are preferred as they will cause less hardware irritation problems in the future. Subtalar joint arthritis is the most common complication. ${ }^{11}$ Osteonecrosis risk is dependent on initial fracture displacement and in open fractures. ${ }^{12}$ Varus malunion due to medial communition ,non-union maybe seen which predispose to development of arthritis. ${ }^{13}$ Skin complications such as skin necrosis, infections, poor wound healing are disastrous complications which should be minimized by proper limb elevation, magnesium sulphate dressing, proper antibiotic use, tension free suturing of the wound. If swelling does not permit surgery, delaying open reduction after temporary stabilization of the fracture either with closed reduction or temporary spanning external fixator maybe required. ${ }^{14} \mathrm{We}$ believe in presence of medial malleolar fracture, approaching the talar fracture through the medial malleolus fracture site may help in reducing the need for another anterolateral incision. Thus, it is an essential tool in such cases and at this site with poor skin condition and poor skin healing. The management of such fractures remains challenging however given their propensity for avascular necrosis, non-union, subtalar arthritis and the optimal management remains to be seen with further studies and newer treatment modalities.

\section{CONCLUSION}

Talus fractures remain a challenge in modern orthopedics. Associated fractures, skin condition issues, poor visualization in standard x-rays along with the high propensity of post operative complications like osteonecrosis, subtalar arthritis, skin necrosis warrant a high index of suspicion as well as prompt management of these cases. We believe that skin condition and fracture displacement are the two most important factors to consider while planning operative management of displaced talus fractures. The presence of associated medial malleolus fractures allows access to the talus through the fracture site and can aid in reduction as well as fixation of this fracture. This can also reduce the need for another anterolateral incision. Further studies and long term follow up is essential to add to the existing data and help decide the optimal management protocol of this fracture.

\section{Funding: No funding sources \\ Conflict of interest: None declared \\ Ethical approval: Not required}

\section{REFERENCES}

1. Canale ST. Fractures of the neck of the talus. Orthopedics. 1990;13:1105-15.

2. Fortin PT, Balazsy JE. Talus fractures: evaluation and treatment. J Am Acad Orthop Surg. 2001;9:114-27.

3. Mechchat A, Bensaad S, Shimi M. Unusual ankle fracture: a case report and literature review. J Clin Orthop Trauma. 2014;5:103-6.

4. Cöster MC, Rosengren BE, Bremander A, Brudin L, Karlsson MK. Comparison of the self-reported foot and ankle score (SEFAS) and the American Orthopedic Foot and Ankle Society score (AOFAS). Foot Ankle Int. 2014;35(10):1031-6. 
5. Buza III JA, Leucht P. Fractures of the talus: Current concepts and new developments. Foot Ankle Surg. 2018;24(4):282-90.

6. Elgafy H, Ebraheim NA, Tile M, Stephen D, Kase J. Fractures of the talus: experience of two level 1 trauma centers. Foot Ankle Int. 2000;21(12):1023-9.

7. Lindvall E, Haidukewych $G$, DiPasquale $T$, Herscovici Jr D, Sanders R. Open reduction and stable fixation of isolated, displaced talar neck and body fractures. JBJS. 2004;86(10):2229-34.

8. Vallier HA, Nork SE, Barei DP, Benirschke SK, Sangeorzan BJ. Talar neck fractures: results and outcomes. JBJS. 2004;86(8):1616-24.

9. Vallier HA, Nork SE, Benirschke SK, Sangeorzan BJ. Surgical treatment of talar body fractures. JBJS. 2003;85(9):1716-24.

10. Van Bergen CJ, Tuijthof GJ, Sierevelt IN, van Dijk CN. Direction of the oblique medial malleolar osteotomy for exposure of the talus. Arch Orthop Trauma Surg. 2011;131(7):893-901.
11. Halvorson JJ, Winter SB, Teasdall RD, Scott AT. Talar neck fractures: a systematic review of the literature. J Foot Ankle Surg. 2013;52(1):56-61.

12. Dodd A, Lefaivre KA. Outcomes of talar neck fractures: a systematic review and meta-analysis. J Orthop Trauma. 2015;29(5):210-5.

13. Sangeorzan BJ, Wagner UA, Harrington RM, Tencer AF. Contact characteristics of the subtalar joint: the effect of talar neck misalignment. J Orthop Res. 1992;10(4):544-51.

14. Chateau PF, Brokaw DS, Jelen BA, Scheid DK, Weber TG. Plate fixation of talar neck fractures: preliminary review of a new technique in twenty-three patients. J Orthop Trauma. 2002;16(4):213-9.

Cite this article as: Katti SN, Paigude PA, Ingale PA, Mane SR. A case of fracture talus with medial malleolus treated with headless screws and cancellous cannulated screws using single anteromedial approach. Int J Res Orthop 2022;8:260-3. 